\title{
Effects of supersymmetric QCD in hadronic Higgs production at next-to-next-to-leading order
}

\author{
Robert V. Harlander* \\ Theory Division, CERN, CH-1211 Geneva 23, Switzerland \\ Matthias Steinhauser ${ }^{\dagger}$ \\ II. Institut für Theoretische Physik, Universität Hamburg, D-22761 Hamburg, Germany
}

(Received 21 August 2003; published 15 December 2003)

\begin{abstract}
An estimate of the next-to-next-to-leading order (NNLO) supersymmetric QCD effects for Higgs production at hadron colliders is given. Assuming an effective gluon-Higgs interaction, these corrections enter only in terms of process-independent, factorizable terms. We argue that the current knowledge of these terms up to NLO is sufficient to derive the NNLO hadronic cross section within the limitations of the standard theoretical uncertainties arising mainly from renormalization and factorization scale variations. The supersymmetric contributions are small with respect to the QCD effects, which means that the NNLO corrections to Higgs production are very similar in the standard model and its minimal supersymmetric extension.
\end{abstract}

DOI: 10.1103/PhysRevD.68.111701

PACS number(s): 14.80.Cp, 12.38.Bx, 12.60.Jv

\section{INTRODUCTION}

Gluon fusion is the dominant production mechanism for Higgs bosons at the CERN Large Hadron Collider (LHC) (for reviews, see Refs. [1,2]). A feature of the gluon fusion process is that it is loop mediated already at leading order. This makes it particularly sensitive to nonstandard particles and couplings as they are predicted by extended theories. A very popular extension of the standard model (SM) is the minimal supersymmetric standard model (MSSM) (for a review, see Ref. [3]), with its five physical Higgs bosons.

We will focus on a scenario where the ratio of the vacuum expectation values of the two Higgs doublets is not too large, $\tan \beta \ll M_{t} / M_{b}$ ( $M_{t}=$ top mass, $M_{b}=$ bottom mass), so that the bottom is much smaller than the top Yukawa coupling. In this case, the dominant effects on the gluon-Higgs coupling in the MSSM arise from the top quark $t$ and its scalar supersymmetric (SUSY) partner, the top squark $\tilde{t}$. SUSY-QCD corrections are induced by virtual gluons $g$ and their fermionic SUSY partners, the gluinos $\tilde{g}$. These effects have recently [4] (see also Ref. [5]) been evaluated at next-toleading order (NLO) in the limit where $M_{\phi}$ $\ll\left\{M_{t}, M_{\tilde{t}}, M_{\tilde{g}}\right\}$, where $\phi$ denotes either of the two $C P$-even Higgs bosons, $h$ or $H$. This limit is expected to work extremely well, if the leading order (LO) dependence on $M_{t}, M_{\tilde{t}}, M_{\tilde{g}}$ is taken into account exactly. This can be inferred from the NLO behavior in the SM [6-9]. In the effective Lagrangian approach, the evaluation of the hadronic Higgs boson cross section factorizes into the calculation of the effective gluon-Higgs boson coupling, times the calculation of the actual process $p p \rightarrow \phi+X$ as mediated by the effective gluon-Higgs boson operator. For a full next-tonext-to-leading order (NNLO) result in this approach, both factors need to be evaluated up to NNLO. However, in the $\mathrm{SM}$, the NNLO contribution of the effective coupling leads

\footnotetext{
*Electronic address: robert.harlander@cern.ch

${ }^{\dagger}$ Electronic address: matthias.steinhauser@desy.de
}

to a numerically negligible contribution, and we will argue that this is true also in the MSSM. The NNLO Higgs boson production cross section can therefore be evaluated from the NLO expression of the effective coupling, as taken from Ref. [4], and the NNLO results for the process diagrams, which are identical to the SM case [10-13].

\section{THE APPROXIMATION}

\section{A. Definition and standard model case}

We use the effective Lagrangian approach where the top quark and all supersymmetric particles are considered heavy with respect to the Higgs boson, see Ref. [4]. In this case, the hadronic cross section $\sigma_{h k} \equiv \sigma(h k \rightarrow \phi+X)$ for Higgs boson production can be written as

$$
\begin{gathered}
\sigma_{h k}(z)=\sigma_{0} C^{2} \Sigma_{h k}(z), \\
\Sigma_{h k}(z)=\sum_{i, j} \int_{z}^{1} \mathrm{~d} x_{1} \int_{z / x_{1}}^{1} \mathrm{~d} x_{2} \varphi_{i / h}\left(x_{1}\right) \varphi_{j / k}\left(x_{2}\right) \hat{\Sigma}_{i j}\left(\frac{z}{x_{1} x_{2}}\right), \\
z \equiv \frac{M_{\phi}^{2}}{s}
\end{gathered}
$$

where $i, j$ denote any partons inside the hadrons $h, k$, and $\varphi_{i / h}(x)$ are the parton densities; $M_{\phi}$ is the Higgs boson mass, and $s$ is the hadronic center-of-mass (c.m.) energy. The coefficient function $C$, defined below, contains the remnant dependence of the gluon-Higgs coupling on the heavy masses, and $\sigma_{0}$ is defined such that the leading order dependence on these masses of $\sigma_{h k}(z)$ is exact. Its exact form is irrelevant for our argument and shall not be given here, owing to space limitations (see, e.g. Ref. [4]).

The partonic expression can be expanded in terms of $\alpha_{s}$,

$$
\hat{\Sigma}_{i j}(x)=\hat{\Sigma}_{i j}^{(0)}(x)+\frac{\alpha_{s}}{\pi} \hat{\Sigma}_{i j}^{(1)}(x)+\left(\frac{\alpha_{s}}{\pi}\right)^{2} \hat{\Sigma}_{i j}^{(2)}(x)+\mathcal{O}\left(\alpha_{s}^{3}\right),
$$



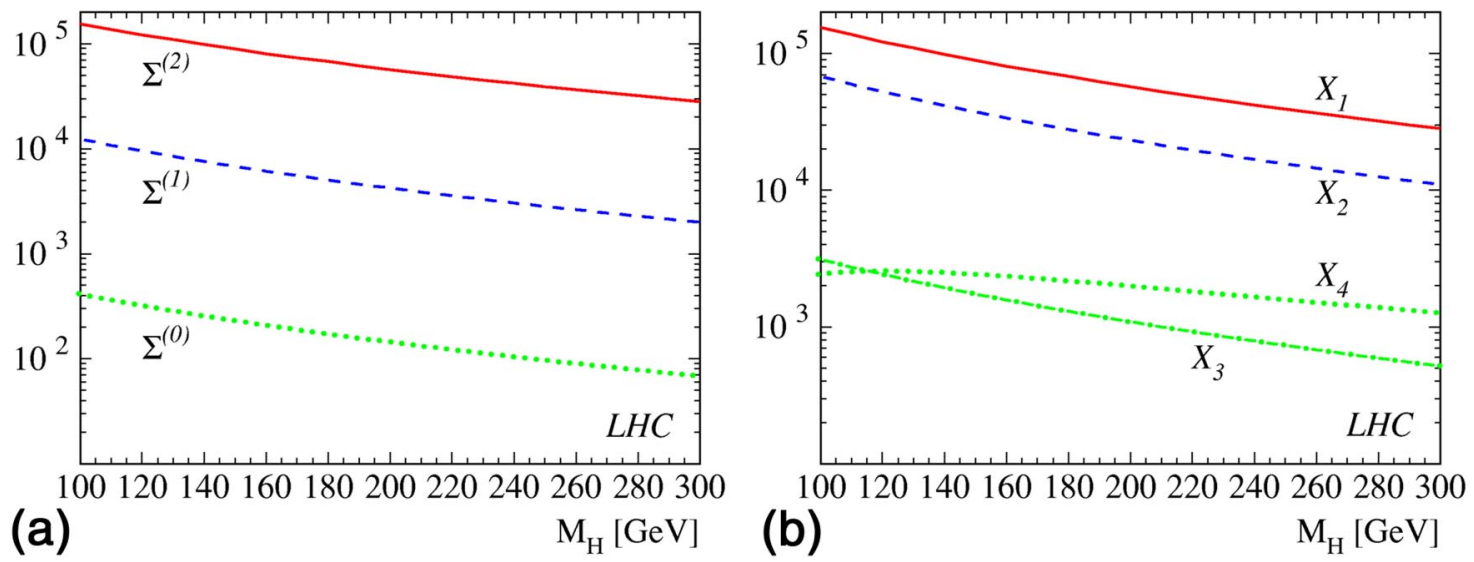

FIG. 1. (Color online) Individual NNLO contributions to the total hadronic Higgs production cross section. The notation is defined in Eqs. (6) and (8). The renormalization and factorization scale $\mu_{\mathrm{R}}$ and $\mu_{\mathrm{F}}$ are identified with the Higgs boson mass $M_{\phi}$.

where $x \equiv M_{\phi}^{2} / \hat{s}$, and $\hat{s}$ is the partonic c.m. energy. Here and in what follows, $\alpha_{s}$ denotes the $\overline{\mathrm{MS}}$-renormalized strong coupling constant for five active quark flavors, evaluated at the renormalization scale $\mu_{\mathrm{R}}$.

For the coefficient function, we write

$$
C\left(\alpha_{s}\right)=\frac{\alpha_{s}}{\pi} C^{(0)}\left[1+\frac{\alpha_{s}}{\pi} \kappa_{1}+\left(\frac{\alpha_{s}}{\pi}\right)^{2} \kappa_{2}+\mathcal{O}\left(\alpha_{s}^{3}\right)\right]
$$

In the SM, the $\kappa_{i}$ are known for $i=1, \ldots, 3[14,15]\left(\kappa_{3}\right.$ contributes only at $\mathrm{N}^{3} \mathrm{LO}$ ). In the MSSM, $\kappa_{1}$ has been evaluated only recently [4]. We now define

$$
\begin{aligned}
\sum_{h k}^{(n)}(z)= & \sum_{i, j} \int_{z}^{1} \mathrm{~d} x_{1} \int_{z / x_{1}}^{1} \mathrm{~d} x_{2} \varphi_{i / h}\left(x_{1}\right) \varphi_{j / k}\left(x_{2}\right) \hat{\Sigma}_{i j}^{(n)}\left(\frac{z}{x_{1} x_{2}}\right), \\
& n \in\{0,1,2\} .
\end{aligned}
$$

For the $\Sigma_{h k}^{(n)}(z), n=0,1,2$, we assume that the parton densities $\varphi_{i / h}$ are evaluated at NNLO. ${ }^{1}$ Thus, the NNLO expression for the hadronic cross section can be written as

$$
\begin{aligned}
\sigma^{\mathrm{NNLO}}= & \sigma_{0}\left(C^{(0)} \frac{\alpha_{s}}{\pi}\right)^{2}\left[\Sigma^{(0)}+\frac{\alpha_{s}}{\pi}\left(\Sigma^{(1)}+2 \kappa_{1} \Sigma^{(0)}\right)\right. \\
& \left.+\left(\frac{\alpha_{s}}{\pi}\right)^{2}\left(\Sigma^{(2)}+2 \kappa_{1} \Sigma^{(1)}+\left(\kappa_{1}^{2}+2 \kappa_{2}\right) \Sigma^{(0)}\right)\right]
\end{aligned}
$$

where the indices $h, k \in\{p, \bar{p}\}$ have been dropped for simplicity. The basis of our estimate of the NNLO terms in SUSY will be that the numerical effect of the term proportional to $\kappa_{2}$ in Eq. (6) is negligible compared to the theoretical uncertainty of the NNLO prediction.

\footnotetext{
${ }^{1}$ We use the approximate NNLO parton densities of Ref. [16].
}

For that purpose, let us look at the relative magnitude of the $\Sigma^{(n)}$ in the case of Higgs boson production at the LHC, Fig. 1(a). We see that $\Sigma^{(0)}$ is more than two orders of magnitude smaller than $\Sigma^{(2)}$, which suggests that the effects from $\kappa_{2}$ can be neglected, if $\kappa_{2}$ is not too large. In order to get a feeling for the magnitude of $\kappa_{2}$, let us look at the SM case. There we have $[14,15]$ :

$$
\begin{aligned}
C^{(0), \mathrm{SM}} & =-\frac{1}{3}, \quad \kappa_{1}^{\mathrm{SM}}=\frac{11}{4}=2.75, \\
\kappa_{2}^{\mathrm{SM}} & =\frac{2777}{288}+\frac{19}{16} l_{\mu t}+n_{f}\left(-\frac{67}{96}+\frac{1}{3} l_{\mu t}\right) \\
n_{f} & =5 \\
& \approx 6.153+2.854 l_{\mu t},
\end{aligned}
$$

with $l_{\mu t} \equiv \ln \left(\mu_{\mathrm{R}}^{2} / M_{t}^{2}\right)$, where $\mu_{\mathrm{R}}$ is the renormalization scale and $M_{t}$ is the on-shell top quark mass.

Using these numbers, we arrive at Fig. 1(b). It shows the relative size of the four terms that contribute to the cross section in Eq. (6) at order $\alpha_{s}^{4}$ :

$$
\begin{aligned}
& X_{1}=\Sigma^{(2)}, \quad X_{2}=2 \kappa_{1} \Sigma^{(1)}, \\
& X_{3}=\kappa_{1}^{2} \Sigma^{(0)}, \quad X_{4}=2 \kappa_{2} \Sigma^{(0)} .
\end{aligned}
$$

As expected from the numerical value of $\kappa_{2}^{\mathrm{SM}}$, Eq. (7), $X_{4}$ is indeed negligible with respect to $X_{1}$ : it is down by a factor of 30 . But another remarkable observation is that the term proportional to $\Sigma^{(1)}$, i.e. $X_{2}$, amounts to around $30 \%$ of the full $\alpha_{s}^{4}$ contribution. For comparison, the $\left(2 \kappa_{1} \Sigma^{(0)}\right)$ term in Eq. (6) amounts to only $15 \%$ of the complete $\alpha_{s}^{3}$ contribution.

To summarize, in the SM, the $\alpha_{s}^{3}$ term $\kappa_{2}$ to the coefficient function of Eq. (4) gives a negligible contribution to the NNLO cross section. In fact, we checked that the difference 
between the true ${ }^{2}$ and the approximate NNLO cross section (i.e. with $\kappa_{2}=0$ ) is less than $1 \%$ at the LHC. This is much smaller than the theoretical uncertainty of around $15 \%$, as estimated by the variation of the factorization and the renormalization scale at NNLO. On the other hand, the knowledge of $\kappa_{1}$ is, relatively speaking, numerically more important for the NNLO than for the NLO contribution to the cross section, for which it was originally evaluated [4].

\section{B. Minimal supersymmetric standard model}

In the MSSM we can parametrize the NLO corrections to the effective Lagrangian as

$$
\kappa_{1}^{\mathrm{SUSY}}=\kappa_{1}^{\mathrm{SM}}+\delta \kappa_{1}=\frac{11}{4}+\delta \kappa_{1} .
$$

In addition, the tree-level normalization of Eq. (4), $C^{(0)}$, changes, of course, but this is irrelevant for our discussion. $\delta \kappa_{1}$ was recently computed in Ref. $[4]^{3}$ and was shown to be negative, with

$$
\left|\delta \kappa_{1}\right| \lesssim 1
$$

for relevant values of the SUSY parameters ${ }^{4}$ (recall that we restrict ourselves to $\left.\tan \beta \ll M_{t} / M_{b}\right)$. It is thus reasonable to assume that also the value of $\kappa_{2}$ in SUSY-QCD will be of the same order of magnitude as in the SM (or smaller). Combining this assumption with the discussion of Fig. 1 (see above) leads us to the conclusion that the NNLO cross section for hadronic Higgs production in supersymmetry should be approximated well by setting $\kappa_{2} \approx \kappa_{2}^{\mathrm{SM}}$.

\section{RESULTS}

As in Ref. [4], we will neglect squark mixing and set the bottom Yukawa coupling to zero for simplicity. More detailed phenomenological studies have to be deferred to a forthcoming publication. Figure 2 shows the NLO and the NNLO $K$ factor, $K_{X} \equiv \sigma^{X} / \sigma^{\mathrm{LO}}(X=\mathrm{NLO}, \mathrm{NNLO})$ in the SM case (dashed), and in the MSSM, for $M_{\tilde{t}}=M_{t}=175 \mathrm{GeV}$, and $M_{\tilde{g}}=500 \mathrm{GeV} ; \sigma^{\mathrm{LO}}, \sigma^{\mathrm{NLO}}$, and $\sigma^{\mathrm{NNLO}}$ are evaluated with LO, NLO, and NNLO parton densities and $\alpha_{s}$ evolution.

The NNLO result in the MSSM is given by the narrow (red) band, arising from the variation of $\kappa_{2}$ between zero and $2 \kappa_{2}^{\mathrm{SM}}$ [see Eq. (7)]. This should serve as an estimate of the theoretical uncertainty induced by the approximation introduced in Sec. II. Within our approximations, the $K$ factor in Fig. 2 is valid for both $C P$-even Higgs bosons of the MSSM,

\footnotetext{
${ }^{2}$ Within the effective theory approach.

${ }^{3}$ In the notation of Ref. [4], it is $c^{\mathrm{SUSY}}=\delta \kappa_{1}+\mathcal{O}\left(\alpha_{s}\right)$.

${ }^{4}$ The expression for $\delta \kappa_{1}$, as presented in Ref. [4], is logarithmically divergent for large ratios among the SUSY particle masses. Such a case requires to resum these logarithms, which is the subject of future studies. It does not affect the arguments of this paper.
}

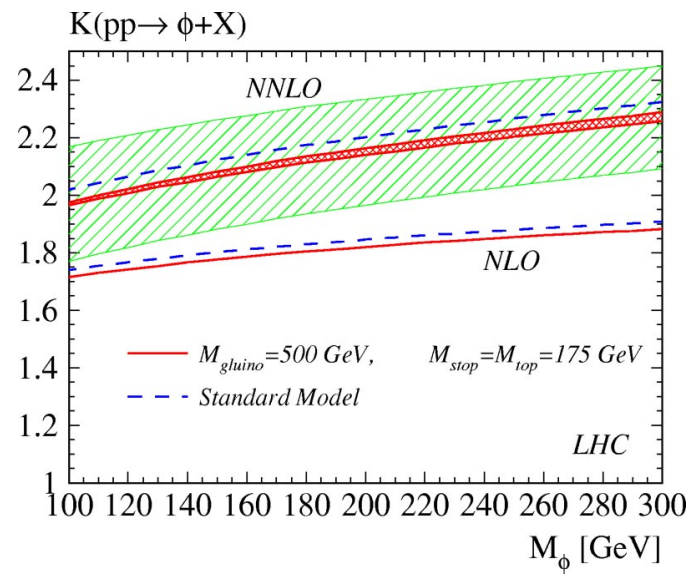

FIG. 2. (Color online) $K$ factor in the standard model (dashed, blue) and the MSSM (solid, red) for the indicated set of parameters. The narrow (red) band in the NNLO MSSM curve corresponds to varying $\kappa_{2}$ between zero and $2 \kappa_{2}^{\mathrm{SM}}$. The renormalization and factorization scales $\left(\mu_{\mathrm{R}}, \mu_{\mathrm{F}}\right)$ have been identified with the Higgs mass in these curves. The diagonally shaded band (green) corresponds to the variation of $\mu_{\mathrm{R}}$ between $2 M_{\phi}$ and $M_{\phi} / 2$ in the NNLO result with $\kappa_{2}=\kappa_{2}^{\mathrm{SM}}\left(\mu_{\mathrm{F}}=M_{\phi}\right)$.

since the Yukawa coupling cancels in the ratio of the LO to the higher order results.

As at NLO, the SUSY effects are small with respect to the QCD effects at NNLO, so that the total $K$ factor in the MSSM is very similar to its SM value. The theoretical uncertainties due to variation of the renormalization and factorization scales are almost identical to the SM case, since the only source of additional scale dependence in the MSSM arises from $\kappa_{2}$. For $\kappa_{2}=\kappa_{2}^{\mathrm{SM}}$, the $\mu_{\mathrm{R}}$ dependence of the NNLO prediction is indicated in Fig. 2 as the diagonally shaded band; the $\mu_{\mathrm{F}}$ dependence is much smaller.

\section{CONCLUSIONS}

We have discussed an approximation for the NNLO contributions to supersymmetric Higgs production in gluon fusion. It was argued that the terms neglected from the full NNLO result should amount to only a few percent, which is much smaller than the theoretical uncertainty induced by the residual renormalization and factorization scale dependence, as well as the anticipated experimental accuracy. For a given set of SUSY parameters, and within the restrictions on these parameters as discussed in the main text, the production cross section for a Higgs boson in the MSSM is thus known to a precision similar to that in the SM. More detailed studies of the MSSM parameter space are clearly desirable and will be presented elsewhere.

\section{ACKNOWLEDGMENT}

We would like to thank T. Plehn for carefully reading the manuscript. 
[1] J.F. Gunion, H.E. Haber, G. Kane, and S. Dawson, The Higgs Hunter's Guide (Addison-Wesley, Reading, MA, 1990).

[2] M. Spira, Fortschr. Phys. 46, 203 (1998).

[3] S.P. Martin, hep-ph/9709356; in "Perspectives on supersymmetry," edited by G.L. Kane, pp. 1-98.

[4] R.V. Harlander and M. Steinhauser, Phys. Lett. B 574, 258 (2003).

[5] S. Dawson, A. Djouadi, and M. Spira, Phys. Rev. Lett. 77, 16 (1996).

[6] S. Dawson, Nucl. Phys. B359, 283 (1991).

[7] A. Djouadi, M. Spira, and P.M. Zerwas, Phys. Lett. B 264, 440 (1991).

[8] D. Graudenz, M. Spira, and P.M. Zerwas, Phys. Rev. Lett. 70, 1372 (1993).
[9] M. Spira, A. Djouadi, D. Graudenz, and P.M. Zerwas, Nucl. Phys. B453, 17 (1995).

[10] R.V. Harlander, Phys. Lett. B 492, 74 (2000).

[11] R.V. Harlander and W.B. Kilgore, Phys. Rev. Lett. 88, 201801 (2002).

[12] C. Anastasiou and K. Melnikov, Nucl. Phys. B646, 220 (2002).

[13] V. Ravindran, J. Smith, and W.L. van Neerven, Nucl. Phys. B665, 325 (2003).

[14] K.G. Chetyrkin, B.A. Kniehl, and M. Steinhauser, Phys. Rev. Lett. 79, 353 (1997); Nucl. Phys. B510, 61 (1998).

[15] M. Krämer, E. Laenen, and M. Spira, Nucl. Phys. B511, 523 (1998).

[16] A.D. Martin, R.G. Roberts, W.J. Stirling, and R.S. Thorne, Eur. Phys. J. C 23, 73 (2002). 